\title{
Virtualização em Redes Terrestre-Satélite 5G
}

\author{
Karine L. M. da Costa ${ }^{1}$, Tibério Tavares Rezende ${ }^{1}$, Antônio Marcos Alberti ${ }^{1}$ \\ ${ }^{1}$ ICT Lab - Instituto Nacional de Telecomunicações (INATEL) \\ Caixa Postal 37.540-000 - Santa Rita do Sapucaí - MG - Brasil \\ \{karine.costa; tiberio\}emtel.inatel.br, alberti@inatel.br
}

\begin{abstract}
In order to propose a hybrid terrestrial-satellite architecture for the $5 G$ infrastructure in Brazil, it is sought to discover and understand the resources and tools used in the simulation or emulation of these network architectures for performance analysis purposes when evaluating various technological interoperability scenarios. Based on the main projects of the European Commission $H 2020$ and standardization organizations of the $5 G$ network, such as 3GPP, ETSI, ITU, among others, this article investigates how the standardization and implementation of $5 G$ terrestrial network management and virtualization technologies are being applied. This article outlines usage scenarios and the tools used in their investigations.
\end{abstract}

Resumo. No intuito de propor uma arquitetura híbrida terrestre-satélite para infraestrutura da rede $5 G$ no Brasil, almeja-se descobrir e entender quais os recursos e ferramentas utilizados na simulação ou emulação dessas arquiteturas de rede para fins de análise de desempenho dos diversos cenários possíveis de interoperabilidade tecnológica. Fundamentado nos principais projetos da Comissão Europeia $H 2020$ e órgãos padronizadores da rede 5G, tais como 3GPP (3rd Generation Partnership Project), ETSI (European Telecommunications Standards Institute), ITU (International Telecommunications Union), dentre outros, este artigo investiga como vêm sendo realizadas a padronização e implementação das tecnologias de virtualização e gerenciamento das redes $5 G$ terrestre-satélite no mundo. Este artigo destaca cenários de uso e as ferramentas utilizadas em suas investigações.

\section{Introdução}

O notável desenvolvimento do ambiente de redes e das tecnologias de comunicação levou a nossa sociedade a um passo mais próximo da comunicação onipresente, em que numerosos dispositivos, por exemplo, computadores pessoais, telefones celulares e equipamentos inteligentes, podem ser conectados à Internet a qualquer momento. Em um futuro próximo, espera-se que, além desses dispositivos, todas as outras coisas físicas do planeta também estejam ligadas à rede [T. Pasca 2017]. Um dos principais diferenciais do sistema de Internet móvel de quinta geração, conhecido como 5G, é sua capacidade de suportar as tecnologias necessárias para a implantação da Internet das Coisas (em inglês, Internet of Things - IoT) [Y. Kawamoto 2013]. Neste contexto, a tecnologia de comunicação via satélite deve ser exposta sempre como uma possibilidade chave, visando atender os mais diversos cenários de uso no 5G. Esse tipo de tecnologia desempenhará um papel importante ao lado das tecnologias terrestres existentes. O uso da comunicação satelital nas tecnologias geoestacionárias (GEO) e não geoestacionárias (NGEO) visando estabelecer 
comunicação entre as redes de acesso, núcleo e de dados no 5G é uma oportunidade única da atual conjuntura de convergência tecnológica exponencial.

É amplamente reconhecido pelos pesquisadores dos sistemas 5G que nenhuma tecnologia única atenderá a todos os requisitos e que a maneira de cumprir os principais indicadores de desempenho $5 \mathrm{G}$ é considerando uma abordagem híbrida de rede. As comunicações via satélite evoluíram para fornecer conectividade contínua e alta taxa de dados com ampla cobertura e alta confiabilidade. Devido suas capacidades inerentes, as comunicações via satélite possuem um papel importante a desempenhar no 5G, o qual está sendo padronizado no 3GPP, com a intenção de ser implementado comercialmente até 2020. O objetivo do 5G é oferecer suporte a vários cenários de uso, como banda larga móvel aprimorada (enhanced Mobile Broadband - eMBB), comunicações ultraconfiáveis e de baixa latência (Ultra Reliable Low Latency Communications - URLLC) e comunicações massivas do tipo de máquina (massive Machine Type Communications mMTC) [3GPP 2016b]. Nesses cenários, as comunicações via satélite serão usadas para ampliar a cobertura de redes terrestres e fornecer backhaul para redes 5G. Os satélites têm outras funções importantes no $5 \mathrm{G}$, incluindo o descarregamento de redes terrestres e o fornecimento de comunicações de emergência confiáveis.

Historicamente, em gerações de redes móveis anteriores, a integração do satcom se baseava em soluções sob medida proprietárias, tanto no nível de rede móvel quanto na comunicação por satélite. Na maioria das vezes, os satélites de telecomunicações eram considerados independentemente das redes terrestres. Nos raros casos em que as soluções híbridas foram propostas, a rede de satélites foi usada principalmente para fornecer backhaul a algumas células individuais remotas e pouco acessíveis como uma rede de transporte simples, não flexível e muito cara. A visão do $5 \mathrm{G}$ em conjunto com satélite na oferta de serviços avançados, como virtualização, capacitação de cidades inteligentes, ambientes inteligentes e comunicação M2M (em inglês, Machine-to-Machine), podem suprir esta demanda [Naser Al-Falahy 2017]. As comunicações por satélite desempenham um papel significativo na implementação do $5 \mathrm{G}$ como uma solução complementar às redes terrestres devido às suas características de cobertura onipresente, broadcast, multicast e recuperação rápida em casos de emergência.

A adoção de tecnologias de virtualização para 5G, como SDN (do inglês, Software Defined Network) e NFV (do inglês, Network Function Virtualization), se mostram como um passo necessário na evolução dos sistemas de segmentos terrestres e de satélite, operando por exemplo, em gateways e terminais de satélite, dentre outros componentes de rede. Esta virtualização permitirá que os serviços de comunicações por satélite sejam entregues de uma forma mais flexível, ágil e eficaz em termos de custos do que é feito hoje, facilitando grandemente a integração e operação sem descontinuidades de redes híbridas. Estes fatores têm sido um dos principais motivadores deste trabalho. Assim sendo, este documento está organizado em 5 Seções. A Seção 2 apresenta as principais atividades de padronização e projetos referentes à comunicação via satélite no contexto 5G. Entendendo que a virtualização é um habilitador potencial para a implementação do 5G, a Seção 3 apresenta um overview das tecnologias de virtualização de rede, SDN e NFV, destacando o estado atual de suas padronizações e como vêm sendo empregadas no cenário terrestre-satélite 5G. Visando contribuir com a padronização $5 \mathrm{G}$ no Brasil, temse como objetivo principal, propor uma arquitetura híbrida terrestre-satélite para a rede 
5G. Para isso, o desenvolvimento de um ambiente de testes para essas redes se faz necessário. Logo, a Seção 4 apresenta o ambiente de testes, cenários de usos e um overview das possíveis ferramentas que serão utilizadas no ambiente de testes SAT-5G em fase inicial de implementação no ICT Lab (Information and Communications Technologies Laboratory), laboratório do Instituto Nacional de Telecomunicações - Inatel. A Seção 5 apresenta as principais conclusões.

\section{Comunicação via Satélite para Atendimento das Redes 5G}

Pretende-se que a rede 5G seja uma rede heterogênea e que torne realidade o conceito de conectividade ubíqua. É possível presenciar um momento de transformação tecnológica no qual há muitos modelos de negócios disruptivos, onde empresas passam a ser plataformas aumentando significativamente a quantidade de dados disponíveis e acessados pelos usuários. O cenário IoT em que qualquer dispositivo pode ser conectado e controlado através da Internet, satélite e redes móveis, traz um aumento significativo na quantidade de dados e dispositivos interligados. A maior dificuldade deste cenário é alcançar a heterogeneidade das diversas redes que formam o universo $5 \mathrm{G}$ e futuramente IoT, pois estão surgindo diversas soluções para essa nova geração de conectividade, mas com diferentes protocolos. Sabe-se que a rede 5G não está sendo projetada para resolver um problema específico, mas sim para ter capacidade de lidar com uma ampla gama de cenários de uso [5G 2016]. Quatro cenários de aplicação têm sido alvo desta pesquisa:

- Wireless Regional Area Network (WRAN): ambiente que exige taxa e latência média da rede. Este cenário foi incluído visando atender países de grandes extensões geográficas como o Brasil.

- Comunicação M2M: aplicações que demandam taxas menores e mais tempo de vida da bateria como rede de sensores, IoT, comunicação veicular e smart places.

- Comunicação com baixa latência: cobre aplicações de Internet Tátil que exigem taxas razoáveis, baixíssima latência e alta confiabilidade [B. Zoran 2016]. Com o padrão de Internet Tátil, as aplicações são executadas em nuvem e o usuário utiliza equipamentos que não processam nenhum dado localmente. Tratase de uma rede ultra-confiável com respostas ultra-rápidas, capaz de entregar experiências táteis remotamente. A abordagem com baixa latência é de grande interesse para a indústria, também por causa dos problemas de segurança de dados [Szymanski 2017].

- Comunicação com alta vazão: abrange cenários como multidões em eventos, ambientes fechados e áreas urbanas densas.

As redes 5G operando juntamente com satélites podem revolucionar a maneira como a Internet atual está construída. O padrão da Internet atual é formado por provedores de serviços de Internet (em inglês, Internet Services Providers - ISP) que estão interligados entre si, juntamente com grandes prestadores de serviços que criam redes menores para conectar os usuários finais como mostrado na Figura 1. Este padrão se mostra inviável para as realizações esperadas com a ubiquidade e com o advento da IoT [A. Siris 2016]. Existem desafios que precisarão ser vencidos, como a alta quantidade de informação que passará a trafegar na rede, uma vez que todo objeto físico terá seu correspondente virtual. 


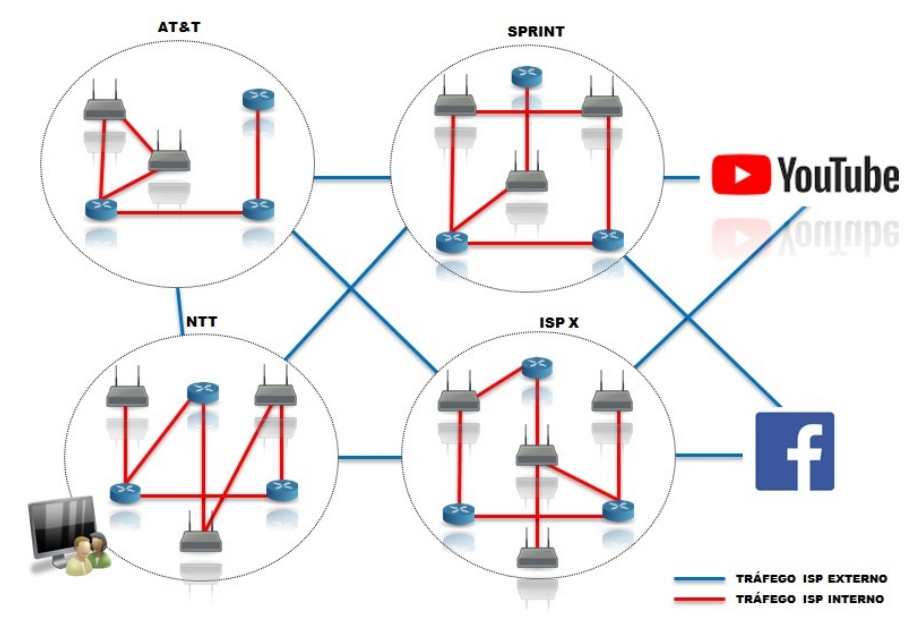

Figura 1. Infraestutura de alto nível da Internet atual.

Embora não tenha sido construído especificamente para IoT, o 5G aparece como um componente chave na camada de comunicação e conectividade da pilha de tecnologias IoT. Promete oferecer confiabilidade, latência, escalabilidade, segurança e mobilidade onipresente que serão exigidos em várias aplicações/serviços de missão crítica no ambiente IoT [Ejaz et al. 2016]. Sua característica de banda larga móvel aprimorada (em inglês, enhanced Mobile Broadband - eMBB) é vista como uma habilitadora da IoT massiva por oferecer capacidade e desempenho de rede em áreas carentes e não atendidas, especialmente em regiões com receita média por usuário baixa (em inglês, Average Revenue per User - ARPU), nos mercados emergentes e em plataformas móveis de aplicações como embarcações e aeronaves [SaT5G 2018].

Os sistemas de comunicação por satélite são a única solução econômica para lidar com esses cenários. Para isso, é necessário que haja a integração perfeita da futura arquitetura 5G e a eficiência ideal seja obtida por meio de colaborações tecnológicas entre os sistemas 5G móveis e satcom. Atualmente, o sistema de comunicação via satélite está passando por uma nova fase de operação com várias renovações de constelação e configuração. O principal caso de uso não está mais construído apenas em torno da voz, como era com a utilização do 2G. A grande diferença com relação as implantações satelitais comerciais anteriores é que estas novas constelações suportam comunicação M2M com foco em IoT. Elas apostam em IoT como uma grande parte de sua estratégia de geração de receita. A tecnologia de satélite serve como um facilitador chave para esses novos serviços, em todas as indústrias, através de fronteiras geográficas para transformar a conectividade IoT como hoje é conhecida [M. De Sanctis 2016].

O principal projeto que trata de satcom para o 5G é o Sat5G [SaT5G 2018]. Este projeto define soluções de backhaul e de descarregamento de tráfego baseadas em satélite. O seu escopo cobre tecnologias $5 \mathrm{G}$ para obter o melhor valor dos recursos de satélites. Por exemplo, multicast para conteúdo e entrega de função de rede virtualizada e atenuação de latência. O projeto também cobre novos modelos de negócios e colaborações operacionais economicamente viáveis que integram as partes interessadas de satélite e terrestres.

Um dos principais objetivos da arquitetura $5 \mathrm{G}$ é um alto grau de flexibilidade para atender melhor às necessidades específicas de campos fora do setor de telecomunicações, 
como por exemplo, indústria automotiva e operação de organizações de missão crítica. Para este tipo de operação a integração da rede $5 \mathrm{G}$ e satélite deverá ser clara e robusta, em particular em relação aos desempenhos de rede e segurança [G. Arfaoui 2018]. Requisitos como QoS de ponta a ponta para esse sistema integrado, segurança e privacidade, como também o uso de vários modelos de confiança, políticas e esquemas de autenticação devem ser explorados visando a rede híbrida. Uma área promissora é a dos sistemas de transporte inteligente (em inglês, Intelligent Transport Systems - ITS), em que a integração de rede 5G e satélite permitirá a implantação em larga escala de ITS, maximizando o desempenho da rede e proporcionando segurança e privacidade aos usuários apesar da grande quantidade de compartilhamento de dados pessoais neste sistema. O projeto 5G CHARISMA [M.C. Parker 2017] pode ser utilizado para aplicações ITS.

Os papéis e vantagens dos satélites em 5G foram estudados pelo 3GPP. A cobertura de satélite traz valores agregados em conjunto com as tecnologias de acesso para 5G, especialmente para aplicações onde a cobertura e a disponibilidade onipresentes são cruciais [S. Parkvall 2017]. O 3GPP agora está padronizando o uso de redes não-terrestres (em inglês, Non-Terrestrial Networks - NTN), isto é, satélites e outros, incluindo veículos aéreos não tripulados, de tal forma que redes de acesso múltiplo podem ser usadas para suportar redes 5G. O primeiro item do estudo NTN começou em fevereiro de 2018 e tem como foco a modelagem de canais para operação das tecnologias. Além da modelagem de canal, as discussões sobre o impacto de NTN do 5G New Radio (NR) [Jin Liu 2016] são realizadas para uma variedade de tópicos como: Random Access Channel (RACH), Hybrid-ARQ (HARQ), Phase Tracking Reference Signal (PTRS), Demodulation Reference Signal (DMRS), Initial Access e Timing Advance.

Com redes de experiência gigabit por segundo com latência controlada e os esperados bilhões de dispositivos conectados à Internet, é clara a necessidade de novas arquiteturas e padrões de sistemas de comunicação. O uso de cargas úteis comumente usadas em satélites pode ser reaproveitado em plataformas próximas à terra. Os serviços de backhaul $5 \mathrm{G}$ podem ser ativados usando essas plataformas próximas à terra que atendem aos requisitos de alta taxa de dados e latência exigidos em 5G. Essas plataformas apresentam vantagens exclusivas como implantação rápida de infraestrutura de banda larga de alta velocidade, cobertura digital em mercados carentes e não atendidos, densificação e implantação de tecnologias de ativação 4G, 5G e IoT, além da maximização da eficiência espectral que permite a oportunidade de melhorar o compartilhamento de espectro.

\section{Virtualização da Rede Terrestre-Satélite 5G}

A visão do $5 \mathrm{G}$ em conjunto com satélite na oferta de serviços avançados, como virtualização, capacitador de cidades inteligentes, ambientes inteligentes e M2M, podem suprir a elevada demanda no tráfego móvel [Naser Al-Falahy 2017]. A adoção de tecnologias de virtualização para 5G, como SDN e NFV, se mostram como passo necessário na evolução dos sistemas de segmentos terrestres e de satélite, operando por exemplo, em gateways e terminais de satélite. Esta virtualização permitirá que os serviços de comunicações por satélite sejam entregues de uma forma mais flexível, ágil e eficaz em termos de custos do que é feito hoje, facilitando a integração e operação sem descontinuidades de redes híbridas.

Em SDNs, o plano de controle é desacoplado do plano de dados e a comunicação 
entre eles é realizada através de APIs (em inglês, Application Programming Interface) (por exemplo, OpenFlow). Em síntese, a arquitetura SDN é composta por três camadas: (1) plano de dados, (2) plano de controle / camada do controlador e (3) aplicação. O plano de dados, baseado em hardware, consiste em dispositivos de encaminhamento (por exemplo, roteadores e comutadores) sendo responsável somente pelo encaminhamento dos dados de acordo com as instruções do controlador. Atualmente, comutadores programáveis estão sendo desenvolvidos usando P4 (em inglês, Programming Protocol-Independent Packet Processors). Atuando como o cérebro, o controlador possui uma visão global e opera toda rede. As necessidades do cliente são abstraídas sobre a camada de aplicação que se comunicam com controlador através das APIs Northbound (por exemplo, API RESTful). Todos os aplicativos / programas são executados acima do controlador. Dentre os controladores mais usados estão: OpenDaylight, Floodlight, NoX / POX, etc. O controlador SDN define a regra para os fluxos de entrada do plano de dados. Camadas SDN se comunicam entre si por meio de APIs abertas chamadas API Northbound Interface (NI) e API Southbound Interface (SI).

$\mathrm{Na}$ tecnologia NFV, as funções de rede são implementadas em componentes de software conhecidas como funções de rede virtual (em inglês, Virtual Network Functions - VNFs). As VNFs são executadas em servidores comuns ou em infraestrutura de nuvem, ao invés de hardwares dedicados, reduzindo o CAPEX (CAPital EXpenditure) e otimizando o provisionamento de recursos. As VNFs podem não apenas ser vinculadas a um serviço e/ou aplicativo específico, mas também podem se relacionar aos requisitos de usuários específicos, políticas de rede e permitir diferenciação de serviços com base nos acordos de nível de serviço (em inglês, Service Level Agreement - SLAs) da assinatura de usuário [Abdelwahab et al. 2016]. A principal motivação é que, uma vez implementadas em software, essas VNFs podem ser executadas em hardware de uso geral, onde podem ser implantadas, configuradas, dimensionadas, migradas e atualizadas sob demanda. Espera-se que a operação das VNFs aconteça de acordo com a estrutura de gerenciamento e orquestração (em inglês, Management and Orchestration - MANO) da NFV definida pelo ETSI.

Analisando as especificações publicadas pelos órgãos padronizadores 3GPP, IETF e ETSI, nota-se que há uma cooperação mútua entre esses órgãos para delinear a utilização das técnicas de SDN e NFV nos sistemas $5 \mathrm{G}$ a fim de oferecer suporte à conectividade de dados e serviços. A especificação TR 32.842 [3GPP 2015] que define o gerenciamento das redes 3GPP, realizada na Release 13, é o primeiro trabalho em conjunto com o ETSI NFV. Nesta especificação, com base no framework arquitetural NFV-MANO do ETSI, estuda-se o gerenciamento do core de redes móveis 3GPP que incluem funções do core de rede virtualizadas. A Figura 2, apresenta o mapeamento do gerenciamento de rede entre 3GPP e ETSI NFV. Baseado nas definições do ETSI, o 3GPP define os elementos de rede (em inglês, Network Element - NE) que não são virtualizados (em inglês, Physical Network Functions - PNF) e aqueles que são virtualizados (VNF). O NFV MANO, conforme definido pelo ETSI NFV, gerencia a infraestrutura virtualizada, as funções de rede virtualizadas (VNF) e os serviços de rede (NS) que abrangem VNFs e PNFs. Na Release 14, o 3GPP expandiu essa abordagem com as especificações 3GPP TS 28.525, 526 e 527 referindo-se as interfaces Os-Ma-nfvo, Ve-Vnfm-em e Ve-Vnfm-vnf. Atualmente, o ETSI NFV está expandindo seu trabalho no ETSI NFV Release 3 para explorar e abordar alguns dos novos casos e requisitos do $5 \mathrm{G}$. 


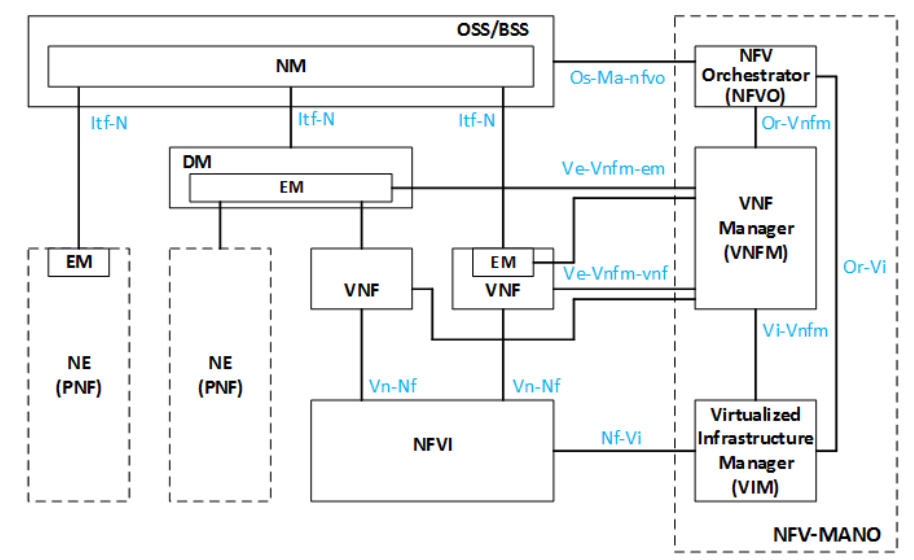

Figura 2. Mapeamento da relação de gerenciamento de rede mista entre o framework arquitetural 3GPP e ETSI NFV-MANO, adaptado de 3GPP TR 32.842 [3GPP 2016a].

Não há especificação ou padronização que engloba a integração SDN/NFV satcom e o $5 \mathrm{G}$ entre os órgãos padronizadores visitados. No entanto, Ferrús et al. [R. Ferrús 2015] descrevem uma arquitetura para sistemas de segmentos terrestres-satélite aptos à SDN/NFV para integração do componente de satélite em sistemas 5G. A Figura 3 refere-se ao modelo de referência de arquitetura para uma rede terrestre-satélite integrada com funções de federação fornecidas pelo 3GPP. Referindo-se ao domínio do operador de rede de satélites (em inglês, Satellite Network Operator - SNO), a parte inferior do modelo é responsável pela gerência dos recursos das infraestruturas de virtualização de função de rede (em inglês, Network Function Virtualization Infrastructure - NFVI) e da conectividade entre NFVIs.

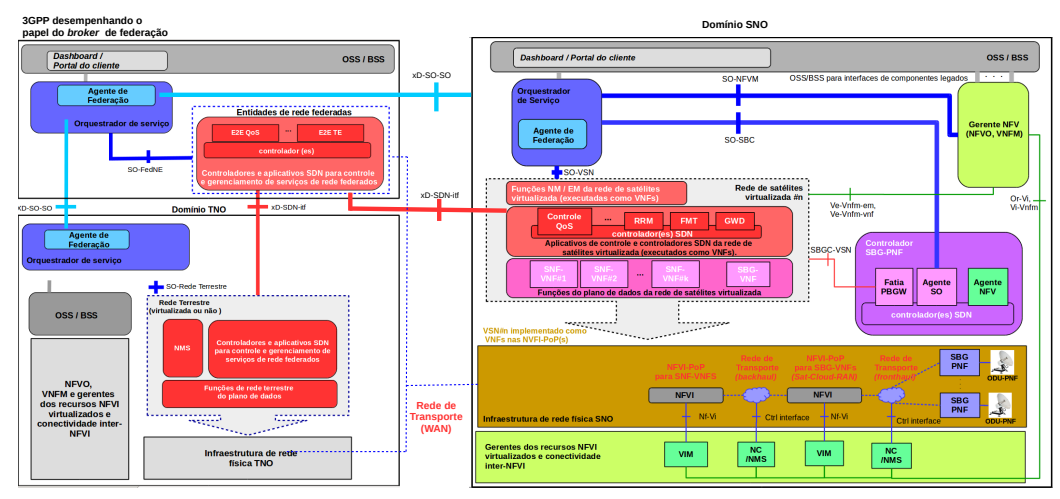

Figura 3. Modelo de referência de arquitetura para uma rede terrestre-satélite integrada com funções de federação fornecidas pelo 3GPP, adaptado de [R. Ferrús 2015].

A infraestrutura de rede física é composta pelos seguintes elementos:

- NFVI-PoPs (NFVI-Points of Presence) para SNF-VNFs (Satellite Network Function-VNFs): fornecendo principalmente recursos de rede, processamento e armazenamento, as NFVI-PoPs executam as SNF-VNFs. Essas infraestruturas não estarão localizadas necessariamente próximas ao $h u b$ do satélite. 
- NFVI-PoP (s) para SBG-VNFs (Satellite Baseband Gateway-VNFs): essa infraestrutura é responsável pela implementação das funções dos gateways de banda base dos satélites. Devido as limitações de distância que podem ser impostas pela rede de fronthaul em termos de latência e largura de banda entre SBG-VNFs e SBGPNFs, provavelmente essas infraestruturas estarão localizadas nas instalações do hub de satélite ou próximas delas.

- SBG-PNFs: conectado diretamente a uma ODU (Out-Door Unit), cada SBG hospeda a parte não virtualizada do gateway de satélite.

- Redes de transporte: a rede backhaul localiza-se entre as NFVI-PoPs e a fronthaul entre as NFVI-PoPs, onde as SBG-VNFs são executadas, e o local que hospeda as SBG-PNFs.

Conforme ilustra a Figura 3, uma ou múltiplas redes de satélite virtualizadas (em inglês, Virtualized Satellite Network - VSN) podem ser estabelecidas. A operação de uma VSN pode ser executada pelo próprio operador de redes de satélite (em inglês, Satellite Network Operator - SNO) ou por outra empresa que exercerá, desse modo, o papel do operador de redes de satélite virtualizadas. Uma VSN é composta pelo plano de dados, pelos controladores e aplicativos de controle e pelos gerenciadores de rede e de elementos. O plano de dados pode ser constituído por uma ou várias SNF-VNFs e SBG-VNFs. Formado por controladores SDN e aplicativos de controle, o plano de controle interage com as VNFs do plano de dados mediante diferentes protocolos. São inúmeras as aplicações de controle que podem ser implementadas, das quais destacam-se: gerenciamento de recursos de rádio (em inglês, Radio Resource Management - RRM), técnicas para mitigação da atenuação (em inglês, Fade Mitigation Techniques - FMT), diversidade de gateway (em inglês, Gateway Diversity - GWD) e controle de QoS. Localizados acima do plano de controle, as funções de NM e EM, podem ser providas como VNFs ou máquinas virtuais (em inglês, Virtual Machines - VMs), fornecendo ao operador de uma determinada VSN um pacote de funções para o gerenciamento da rede de satélites (por exemplo, gerenciamento FCAPS (em inglês, Fault, Configuration, Accounting, Performance and Security)).

\section{Rede Terrestre-Satélite 5G Virtualizada - Hands on}

Pretende-se desenvolver um ambiente de testes ICT SAT-5G no ICT Lab. do Inatel, conforme demonstra a Figura 7, de modo que a equipe de pesquisa possa realizar testes de diferentes configurações da arquitetura terrestre-satélite 5G com a finalidade de encontrar e escolher as melhores ferramentas para modelar as redes satelitais nas concepções GEO e NGEO. Visando contribuir com a padronização 5G no Brasil, tem-se como objetivo principal de pesquisa, propor uma arquitetura híbrida terrestre-satélite para a rede 5G. Para isso, o desenvolvimento de um ambiente de testes para essas redes se faz necessário. Logo, baseado em resultados obtidos em trabalhos anteriores, esta Seção apresenta a configuração para a implementação inicial do ambiente de testes e um overview das possíveis ferramentas que serão utilizadas para investigar os seguintes cenários de uso:

- Cenário de uso 1 - Entroncamento IoT: constituído por uma aplicação IoT implementada em uma Escola Rural de Santa Rita do Sapucaí, este cenário refere-se a uma horta inteligente conectada à Internet, que realiza o controle e gerenciamento do processo de irrigação de seus canteiros. A ideia é demonstrar a vi- 
abilidade da oferta de serviços IoT-5G em áreas rurais utilizando o satélite no entroncamento das rede, conforme ilustra a Figura 4.
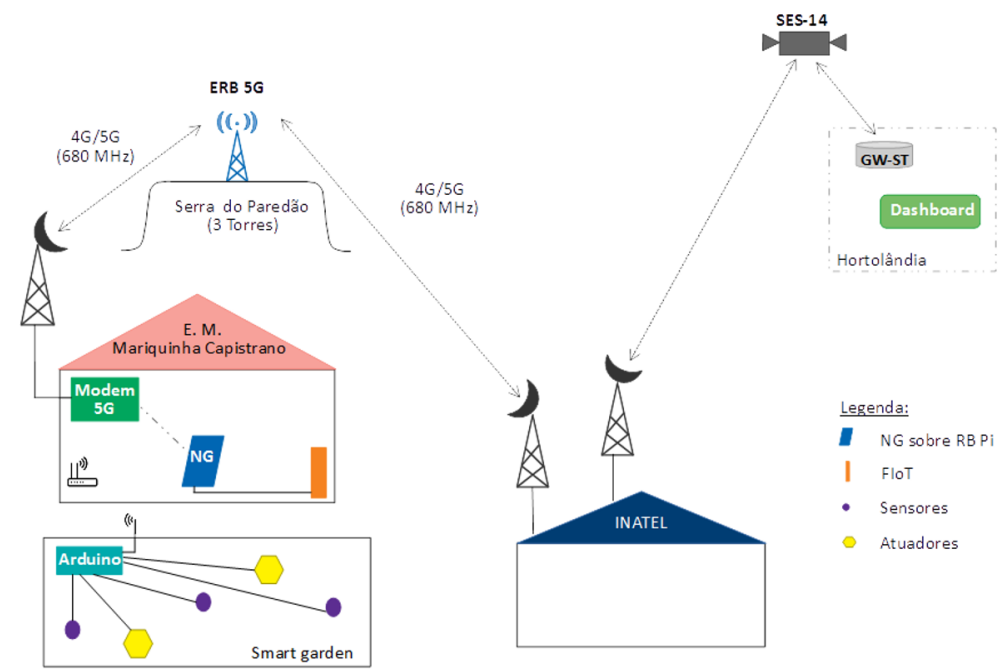

Figura 4. Aplicação loT-5G rural utilizando o satélite no entroncamento das redes.

- Cenário de uso 2 - Teste visando distribuição de conteúdo (Content Delivery Network - CDN e Integrated Delivery Network - IDN) considerando Caching: utilizar caches e outras ferramentas open source para rede híbrida 5G. Avaliar o suporte ao gerenciamento de cache (e streaming adaptativo, se possível) para distribuição de conteúdo. Trabalhos futuros visam testes de cache com arquitetura de Internet do Futuro NovaGenesis, uma rede ICN (em inglês, Information Centric Networking), através do enlace de satélite até o ponto final da rede para comparações.

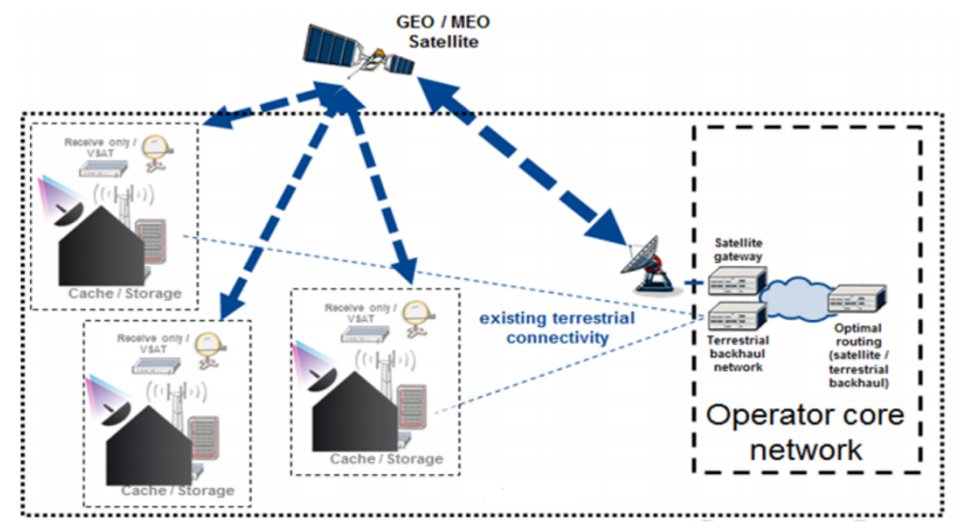

Figura 5. Cenário para testes visando distribuição de conteúdo (CDN) considerando caching. Fonte: Use cases and scenarios of $5 \mathrm{G}$ integrated satellite-terrestrial networks for enhanced mobile broadband [Konstantinos Liolis 2018].

- Cenário de uso 3 - Virtualização de funções de rede e compatibilidade com redes definidas por software (SDN): uma rede terrestre-satélite integrada definida 
por software pode ser divida em três planos: dados, controle e gerenciamento, conforme ilustra a Figura 6. Responsável pelo encaminhamento dos dados, o plano de dados compõe a camada de infraestrutura e é constituído pelos dispositivos terrestres e satélites, e switches Openflow. Os controladores SDN fazem parte do plano de controle, responsáveis pelo gerenciamento e controle da rede SDN. O plano de gerenciamento é composto pelas funções de rede, sendo elas virtuais ou físicas. $\mathrm{O}$ objetivo é inserir o satélite no enlace entre controlador e controlado, avaliando o desempenho em termos de atraso, perda de pacotes, etc.

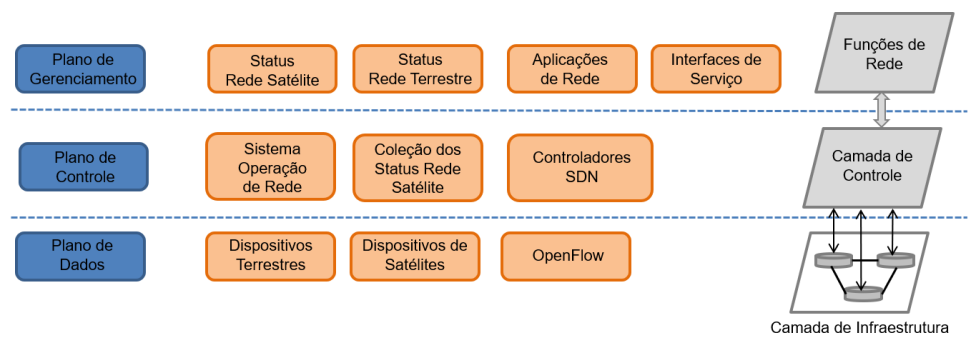

Figura 6. Estrutura funcional de uma rede terrestre-satélite integrada definida por software.

A Figura 7 apresenta a configuração inicialmente desenvolvida para a construção do ambiente de testes ICT SAT-5G, incluindo as plataformas e ferramentas mencionadas abaixo. As ferramentas serão utilizadas nos cenários apresentados para realização de testes, tais como: conectividade IoT e 5G, distribuição de conteúdo de maneira mais eficiente com o uso de satélites e criação de redes virtuais orquestradas por SDN minimizando a latência da rede. Este trabalho apresenta as principais plataformas e ferramentas selecionadas, que vêm sendo integradas na construção do cenário híbrido 5G terrestre-satélite utilizando as tecnologias SDN, NFV, CDN, IDN e ICN:

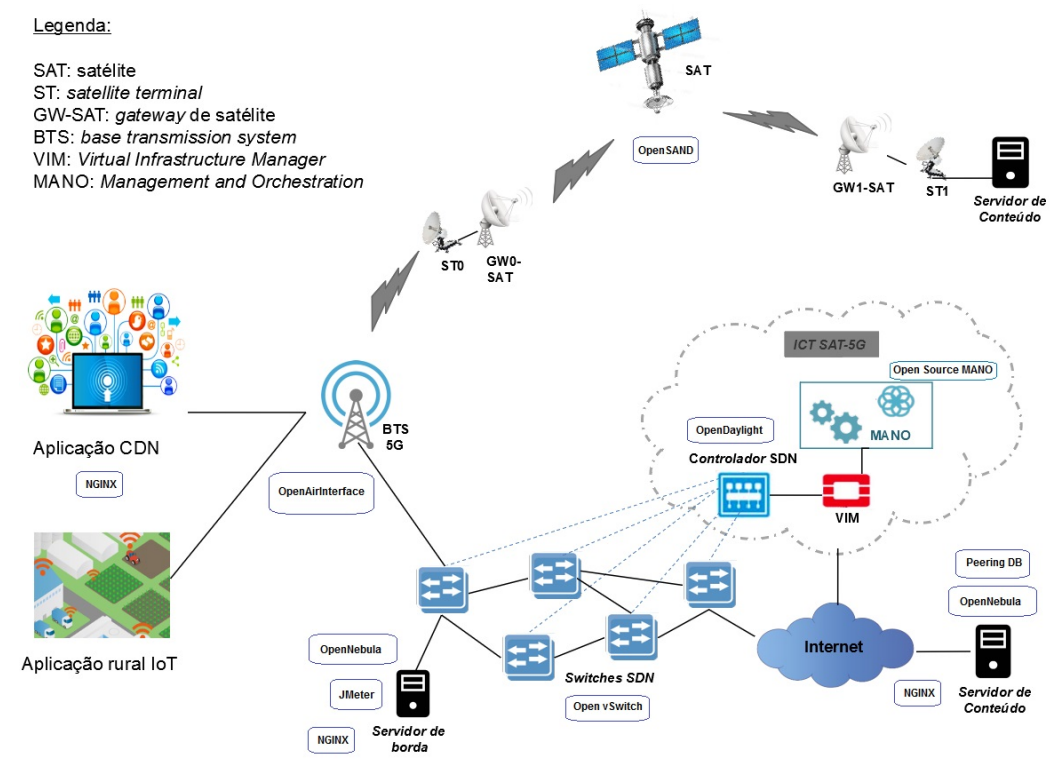

Figura 7. Configuração do ambiente de testes ICT SAT-5G para análises e simulações de arquiteturas de redes terrestre-satélite 5G. 
- OpenSAND: ferramenta mais empregada na virtualização dos componentes de uma rede de satélite, o OpenSAND é uma plataforma open source inspirada nos padrões DVB-S2 / RCS2, capaz de emular as principais características de um sistema satcom. Suporta conectividade IPv4, IPv6 e Ethernet e pode ser interconectada com equipamentos reais e outras redes baseadas em IP (terrestre e/ou satélite), ou até mesmo com o backbone da Internet. Além de fornecer ferramentas de configuração e monitoramento (em tempo real e off-line), permitindo avaliar o desempenho dos cenários emulados.

- OpenDaylight: é uma plataforma SDN. Suporta os principais protocolos utilizados em SDN: OpenFlow, OVSDB, NETCONF, BGP e outros. Com uma estrutura modular, permite que a seleção dos recursos que serão utilizados seja feita pelo usuário, possibilitando a criação de um controlador personalizado.

- Open Source MANO (OSM): baseado no framework definido pelo ETSI para o gerenciamento e orquestração de todos os recursos no data center em nuvem, o OSM é uma comunidade open source que fornece uma pilha MANO para NFV. Esta ferramenta é independente de VIM (do inglês, Virtualized Infrastructure Manager) e adequada a todas as funções de rede virtualizadas.

- Open vSwitch: é uma ferramenta utilizada para a criação de switches virtuais [OvS 2019]. Através desta ferramenta é possível criar múltiplos switches para a automação da rede através de programação. Nos switches criados com o Open vSwitch é possível implementar diversos protocolos, entre eles o OpenFlow, e também definir um controlador como o POX (plataforma de desenvolvimento de software livre para um controlador SDN baseado em Python), o NOX (sistema operacional para a rede), ou o próprio controlador nativo do Open vSwitch [OvS 2019]. O Open vSwitch permite a criação de switches virtuais em máquinas físicas diferentes, similar ao software da VMware. Desta forma é possível criar diversas redes virtuais para testes.

- OpenAirInterface: A missão da OpenAirInterface software Alliance (OSA) é fornecer software e ferramentas para pesquisa e desenvolvimento de produtos sem fio 5G [OpenAirInterface 2019]. A atual geração de hardware e software para a rede de acesso por rádio (RAN) consiste em um grande número de elementos proprietários que sufocam a inovação e aumentam o custo para as operadoras implantarem novos serviços em redes celulares em constante mudança. software de código aberto executado em processadores de uso geral (como x86, ARM) pode simplificar muito o acesso à rede, reduzir custos, aumentar a flexibilidade, aumentar a velocidade de inovação e acelerar o lançamento de novos serviços. A implementação em código aberto de pilha totalmente em tempo real (eNB, UE e rede principal) em processadores de propósito geral quando combinados com SDN, NFV e OpenStack e trazem eficiência significativa no design de RAN tanto de inovação quanto de custo.

- OpenNebula: O OpenNebula é uma solução de código aberto simples, porém poderosa e flexível, para criar Nuvens Privadas e gerenciar a virtualização de Data Centers [OpenNebula 2019]. O OpenNebula fornece recursos nas duas principais camadas de virtualização de data center e infraestrutura de nuvem. O Gerenciamento de Virtualização de Data Center integra-se diretamente a hipervisores (como o KVM) e tem controle total sobre recursos físicos e virtuais, fornecendo recursos avançados para gerenciamento de capacidade, otimização de recursos, 
alta disponibilidade e continuidade de negócios. O Gerenciamento de Nuvem para fornecer uma camada de provisionamento multilocatária semelhante a uma nuvem sobre uma solução de gerenciamento de infraestrutura existente (como o VMware vCenter).

- JMeter: JMeter é uma ferramenta opensource utilizada para testes de carga. Esta ferramenta é parte do projeto Jakarta da Apache software Foundation [JMeter 2019]. O JMeter foi desenvolvido para criar e executar testes de carga em serviços computacionais. Neste projeto de rede terrestre-satélite 5G virtualizada, pode ser usado para testar o desempenho em recursos estáticos e dinâmicos, e também aplicativos dinâmicos da Web. Pode ser usado para simular cargas em um servidor, grupo de servidores e rede ou para analisar o desempenho geral sob diferentes tipos de utilizações de carga [JMeter 2019].

- Peering DB: é um projeto que tem o objetivo de facilitar a troca de informações relacionadas a peering (acordos de troca de tráfego) e conta com o apoio dos principais ASNs e IXPs (Internet Exchange Points) do mundo. Trata-se de um registro de organizações e Sistemas Autonômos em banco de dados com informações dos locais em que fazem troca de tráfego, e qual a política de peering que possuem. Facilidades para o usuário: Gestão mais simples com uma única conta para network, facility e exchange; Um único usuário pode gerenciar múltiplas organizações; Possibilidade de gerenciamento de usuários e atribuição de níveis de acesso.

- NGINX: é uma ferramente utilizada como servidor HTTP, IMAP, POP3, Proxy e também para criação de redes CDN. Em termos práticos, pode ser descrito como um software que processa as solicitações dos usuários da rede garantindo que a troca de informações seja efetuada. Nginx funciona com o método EDA (Eventdriven Architecture), que é preparado para lidar com uma maior demanda de conexões se comparado ao modo convencional, como é o caso do Apache, no qual o processamento é baseado em um único segmento para cada usuário. No Nginx, todo o caminho operado pela conexão de rede é non-blocking. O ponto a destacar é que este servidor se mostra eficiente por consumir poucos recursos da rede. Algumas interfaces de socket são bloqueadas em determinadas situações, enquanto outras retornam o resultado de imediato [NGINX 2019].

Diversas ferramentas e plataformas de virtualização e gerenciamento de redes existentes no mercado têm viabilizado o desenvolvimento de diferentes soluções no que refere-se a arquitetura híbrida terrestre-satélite. A ferramenta OpenSAND é utilizada para simulação da rede de satélite real, e as configurações SDN operam através da OpenDaylight. A orquestração dos recursos da rede é realizada pela OSM. Essa rede híbrida pode conter vários switches criados pela ferramenta Open vSwitch. Já, a OpenAirInterface desenvolve códigos abertos para controle de Radio Access Network (RAN). As ferramentas voltadas para distribuição de conteúdo com cache de rede são: NGINX para criação de uma rede CDN cloud e edge operando com o OpenNebula (gerenciador de nuvens privadas ou públicas), a JMeter e PeeringDB para testes de carga da rede e controle de informações de vários usuários diferentes.

\section{Conclusão}

Percebe-se que o 5G está sendo construído a partir da convergência terrestre/satélite. Isso significa que não existe mais $5 \mathrm{G}$ ou satélite, mas sim $5 \mathrm{G}$ operando através da rede via 
satélite. A arquitetura híbrida é a única que faz sentido no contexto da evolução tecnológica. Para tanto, a padronização do $5 \mathrm{G}$ deve contemplar a arquitetura híbrida na sua totalidade. A interoperabilidade deve ser garantida via padrões amplos e que integrem o enlace satelital ao 5G, sem amarras ou remendos. Diversas ferramentas e plataformas de virtualização e gerenciamento de redes existentes no mercado têm viabilizado o desenvolvimento de diferentes soluções no que refere-se a arquitetura híbrida terrestresatélite. Desse modo, este artigo destaca as principais ferramentas que podem ser integradas para realizar cenários de avaliação híbrido 5G terrestre-satélite. Atualmente o cenário de telecomunicações é de transição, com mais perguntas do que respostas. $\mathrm{O}$ 5G é uma oportunidade única para a convergência terrestre/satélite e para a evolução das comunicações globais. A virtualização das funções da rede para broadcast faz sentido pela otimização da rede, pela visibilidade da rede em relação ao gerenciamento e pela orquestração de forma eficiente. Sendo assim, como próximo trabalho, pretende-se explorar as ferramentas apresentadas neste artigo na implementação de uma rede terrestresatélite 5G definida por software para distribuição de conteúdo.

\section{Agradecimentos}

Este trabalho foi parcialmente financiado pelo Sindicato Nacional das Empresas de Telecomunicações por Satélite (SINDISAT). Também foi parcialmente financiado pela RNP, com recursos do MCTIC, processo No 01250.075413/2018-04, sob o projeto Centro de Referência em Radiocomunicações (CRR) do Instituto Nacional de Telecomunicações - Inatel, Brasil. Os autores agradecem também a FAPEMIG, CNPq e CAPES.

\section{Referências}

3GPP (2015). Telecommunication management; study on network management of virtualized networks. In Release 13.

3GPP (2016a). Tr 32.842 study on network management of virtualized networks. In $3 G P P$.

3GPP (2016b). Tr 38.913 study on scenarios and requirements for next generation access technologies. In Release 14. [Online: 12/12/2015] Disponível em: http://www.3gpp.org/DynaReport/38913.htm Acesso em: 19/07/2018 às 13:40.

5G, A. (2016). 5g americas white papers. http://www.5gamericas.org/ptbr/resources/white-papers/, Novembro.

A. Siris, Y. Thomas, G. P. (2016). Supporting the iot over integrated satellite-terrestrial networks using information-centric networking. In 8th IFIP Int. Conf. New Tech. Mobi. Secur. NTMS 2016, volume 16, pages 03-07.

Abdelwahab, S., Hamdaoui, B., Guizani, M., and Znati, T. (2016). Network function virtualization in 5g. IEEE Communications Magazine, 54(4):84-91.

B. Zoran, B. Bakmaz, M. B. (2016). Vision and enabling technologies of tactile internet realization. In Adv. Tech., Sys. and Ser. in Teleco. (TELSIKS), volume 16, pages 01-07.

Ejaz, W., Anpalagan, A., Imran, M. A., Jo, M., Naeem, M., Qaisar, S. B., and Wang, W. (2016). Internet of things (iot) in 5g wireless communications. IEEE Access, 4:1031010314. 
G. Arfaoui, P. Bisson, R. B. R. B. H. E. E. F. F. K. P. K. N. M. N. P. O. J. P. J. S. M. S. J.-P. W. e. A. Z. (2018). A security architecture for $5 \mathrm{~g}$ networks. In IEEE Access, volume 06, pages 22466-22479.

Jin Liu, S. Sharma, K. S. (2016). Initial access, mobility, and user-centric multi-beam operation in $5 \mathrm{~g}$ new radio. In IEEE Communications Magazine, volume 18, pages $68-84$.

JMeter, A. (2019). In JMeter. [Online]: Disponível em: https://jmeter.apache.org. Acesso em: 26/03/2019 às 11:21.

Konstantinos Liolis, Alexander Geurtz, R. S. N. C. (2018). Use cases and scenarios of $5 \mathrm{~g}$ integrated satellite-terrestrial networks for enhanced mobile broadband: The sat $5 \mathrm{~g}$ approach. International Journal of Satellite Communications and Networking, Maio.

M. De Sanctis, E. Cianca, G. A. I. B. R. P. (2016). Satellite communications supporting internet of remote things. In IEEE Int. of Th. J., volume 03, pages 113-123.

M.C. Parker, G. Koczian, S. W. K. H. V. J. T. R. I. N. M. S. P. K. e. P. J. (2017). Ultra-low latency $5 \mathrm{~g}$ charisma architecture for secure intelligent transportation verticals. In 2017 19th International Conference on Transparent Optical Networks (ICTON), volume 06.

Naser Al-Falahy, O. Y. A. (2017). Technologies for 5g networks: Challenges and opportunities. In IEEE IT Professional, volume 19.

NGINX (2019). [Online]: Ninx. Disponível em: https://www.nginx.com/resources/wiki/. Acesso em: 29/03/2019 às 14:20.

OpenAirInterface, S. A. (2019). In Open Air Interface. [Online]: Disponível em: https://www.openairinterface.org/. Acesso em: 28/03/2019 às 17:07.

OpenNebula, P. (2019). In OpenNebula. [Online]: Disponível em: https://opennebula.org/key-features/. Acesso em: 01/04/2019 às 13:45.

OvS, L. F. (2019). In OpenvSwitch. [Online]: 01/11/2017 Disponível em: https://www.openvswitch.org/. Acesso em: 25/03/2019 às 16:44.

R. Ferrús, H. Koumaras, O. S. e. a. (2015). Sdn/nfv-enabled satellite communications: Ground segment network architecture for 5g integration. Physical Communication.

S. Parkvall, E. Dahlman, A. F. M. F. (2017). Nr: The new 5g radio access technology. In IEEE Communications Standards Magazine, volume 1, pages 68-84.

SaT5G, H. (2018). Sat5g project h2020. http://sat5g-project.eu, Junho.

Szymanski, T. (2017). Strengthening security and privacy in an ultra-dense green $5 \mathrm{~g}$ radio access network for the industrial and tactile internet of things. Wire. Commun. and Mob. Comp. Conf. (IWCMC), page 01-08.

T. Pasca, S. Dama, V. S. K. K. (2017). A feasible cellular internet of things. In IEEE Consum. Elect. Mag., volume 16, pages 66-72.

Y. Kawamoto, H. Nishiyama, Z. M. F. (2013). Effective data collection via satelliterouted sensor system (srss) to realize global-scaled internet of things. In IEEE Sens. $J$. , volume 13, page 3645-3654. 